\title{
Sensitivity of the acoustics of short circular holes with bias flow to inlet edge geometries
}

\author{
Juan Guzmán-Iñigo *, Dong Yang ${ }^{\dagger}$, Holly G. Johnson * and Aimee S. Morgans $\S$ \\ Imperial College London, London SW7 2AZ, United Kingdom
}

\begin{abstract}
Short circular holes with a mean bias flow passing through them can absorb or generate acoustic energy depending on frequency. A recently proposed semi-analytical model (Yang \& Morgans, Journal of Sound and Vibration, 384 (2016) pp. 294-311) based on the Green's function method successfully captured this acoustic absorption and generation. The model pointed to the importance of accurately capturing the path followed by the unsteady vorticity shed from the hole inlet edge. In the present work, we systematically study the effect of the path of the shed vorticity on the hole acoustics. We combine the above model with CFD tools for capturing how the path of the shed vorticity varies for small modifications in the hole inlet edge shape. A chamfered edge, a rounded edge and two elliptical edge cases are considered to show that a very small change to the shape of the hole inlet edge can give rise to significant differences in the hole acoustic response.
\end{abstract}

\section{Nomenclature}

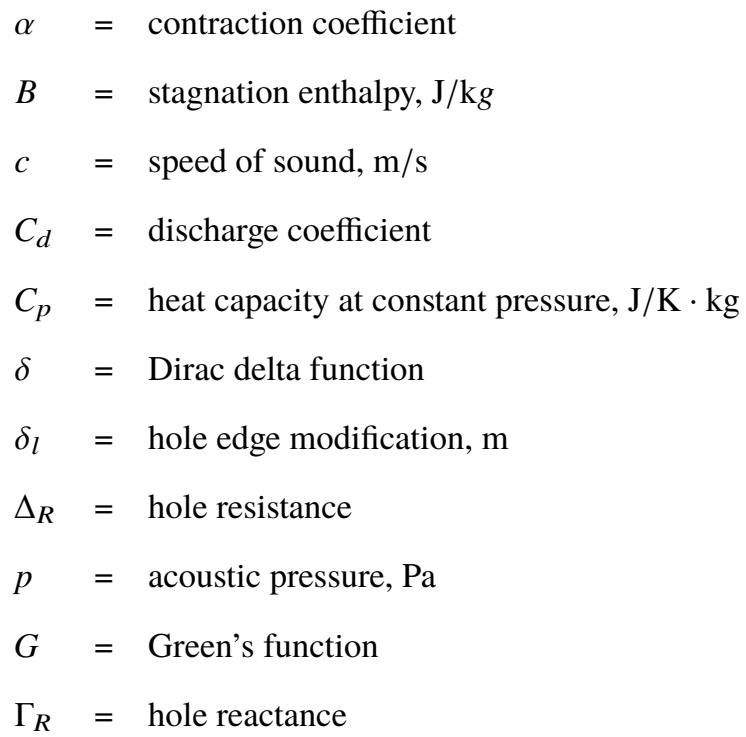

\footnotetext{
*Research Associate, Department of Mechanical Engineering

${ }^{\dagger}$ Research Associate, Department of Mechanical Engineering (Corresponding Author)

${ }^{\ddagger}$ Research Associate, Department of Mechanical Engineering

${ }^{\S}$ Professor, Department of Mechanical Engineering
} 


$$
\begin{aligned}
& H=\text { Heaviside function } \\
& I=\text { turbulence intensity } \\
& k=\text { acoustic wavenumber, } \mathrm{m}^{-1} \\
& k_{0}=\text { vorticity wavenumber, } \mathrm{m}^{-1} \\
& K_{R}=\text { Rayleigh conductivity, } \mathrm{m} \\
& M=\text { Mach number } \\
& \text { n }=\text { outwards normal direction } \\
& L_{h} \quad=\text { hole length, } \mathrm{m} \\
& \lambda=\text { hole opening expansion ratio } \\
& \Pi=\text { acoustic energy absorbed by the hole, } \mathrm{J} / \mathrm{s} \\
& Q=\text { volume flux, } \mathrm{m}^{3} / \mathrm{s} \\
& \text { Re }=\text { Reynolds number } \\
& R_{h}=\text { hole radius, } \mathrm{m} \\
& R_{V}=\text { radial location of the vortex sheet, } \mathrm{m} \\
& \rho=\text { density, } \mathrm{kg} / \mathrm{m}^{3} \\
& s \quad=\quad \text { vortex sheet path, } \mathrm{m} \\
& S=\text { surface, } \mathrm{m}^{2} \\
& S_{t} \quad=\quad \text { Strouhal number } \\
& \sigma \quad=\quad \text { vorticity strength, } \mathrm{s}^{-1} \\
& t=\text { time, } \mathrm{s} \\
& T=\text { temperature, } \mathrm{K} \\
& \mathbf{u}=\text { velocity, } \mathrm{m} / \mathrm{s} \\
& \boldsymbol{u}_{\boldsymbol{c}}=\text { vortex convection velocity, } \mathrm{m} / \mathrm{s} \\
& \bar{u}_{\sigma}=\text { mean flow velocity magnitude at the vena contracta, } \mathrm{m} / \mathrm{s} \\
& V=\text { volume, } \mathrm{m}^{3} \\
& \omega=\text { vorticity, } \mathrm{s}^{-1} \\
& \omega=\text { angular frequency, } \mathrm{rad} / \mathrm{s} \\
& \boldsymbol{x}=\text { coordinates of the physical space, } \mathrm{m} \\
& \hat{x}=\text { normalized axial location of the vortex sheet } \\
& \boldsymbol{y}=\text { coordinates of the acoustic source, } \mathrm{m} \\
& \hat{y}=\text { normalized radial location of the vortex sheet }
\end{aligned}
$$

Superscripts 


$$
\begin{aligned}
\prime \quad & =\text { time-domain perturbation } \\
\sim \quad & =\text { frequency-domain perturbation } \\
-\quad & =\text { steady mean }
\end{aligned}
$$

\section{Subscripts}

$u=$ region upstream of the hole

$d=$ region downstream of the hole

\section{Introduction}

The sound absorption ("damping") or generation ("whistling”) of a short circular hole is relevant to many applications. Many practical situations involve a low Mach number mean flow passing through the holes, such as a cooling flow passing through perforated liners or Helmholtz resonators in aero-engine or power gas turbine combustors [1-6], fuel-air mixtures passing through injectors into combustors [7], and air from the lungs passing through the glottis [8].

At low frequencies, significant acoustic damping occurs due to the transfer of acoustic energy to kinetic energy. When incident acoustic waves interact with the shear layers at the rims of a hole, unsteady vorticity is shed at the hole lip. At high Reynolds numbers, this unsteady vorticity is convected through the hole by the mean flow and eventually dissipated by turbulence far downstream. Theoretical models for hole acoustics usually assume that the vorticity shed at the hole inlet edge is convected downstream by the mean flow to form a thin vortex sheet within the mean shear layer [9]. For an infinitely short circular hole with half spaces either side, Howe [10] assumed a downstream cylindrical vortex sheet with the same radius as the hole, neglected any shear layer instability, and then developed an analytical model which captures the acoustic dissipation well for very short holes [1, 11-18].

When the hole length is of the same order as the hole radius but not long enough to allow the separated mean inlet flow to reattach within it (as shown in Fig. 1), the hole impedance was experimentally found to differ substantially from Howe's predictions [17]. Such holes were found to have the potential to generate as well as absorb acoustic energy, even in the low Strouhal number region. Recent semi-analytical models [19, 20] which account for the vortex-sound interactions both after and within the short hole revealed the importance of accurately capturing the path of the shed vorticity (or the vortex sheet shape). When the vortex path is captured accurately, the acoustic predictions agree well with previous experimental and CFD results [17, 21], for example predicting both the absorption and generation of acoustics.

However, it is widely known that both the flow contraction coefficient and the details of the vortex sheet shape near the hole inlet edge can be sensitive to the hole inlet edge geometry [22]. These changes lead, in turn, to changes in the acoustic response (as shown in experiments [23-26] and numerical simulations [27]). This inspires us to modify (and eventually optimize) the hole acoustics by slightly modifying the hole inlet edge geometries. In the present work, the 


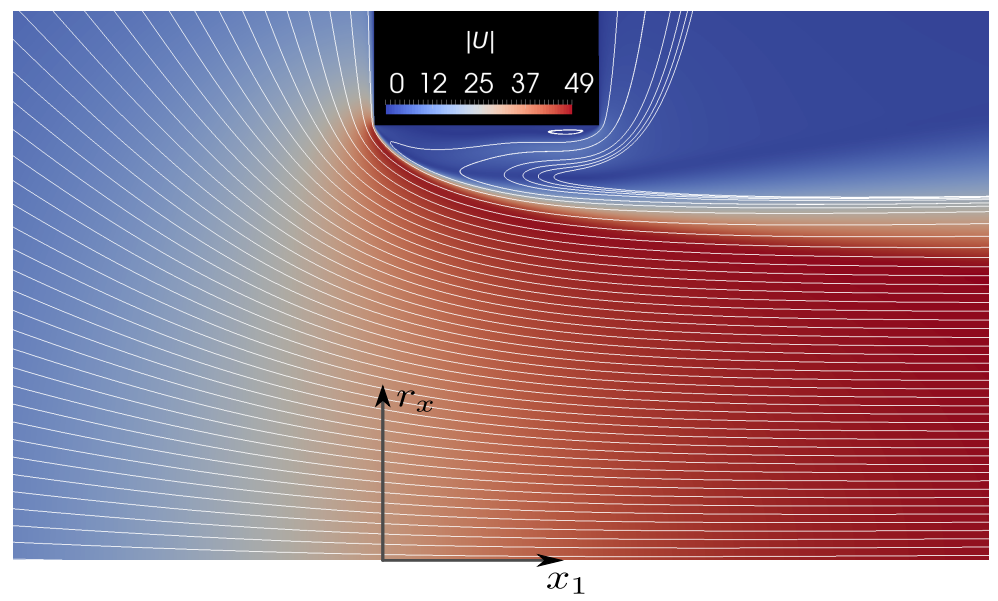

Fig. 1 Mean flow velocity magnitude $|\overline{\mathbf{u}}|$ for a straight-edge hole with $L_{h} / R_{h}=0.5$. White lines denote streamlines. The domain is axisymmetric with $x_{1}$ being the axis of symmetry.

semi-analytical model developed in [19, 20] is firstly used to systematically study the effect of the vortex sheet shape on the hole acoustics. Then, as the vortex sheet shapes are assumed unaffected by small acoustic perturbations [17], the vortex sheet shapes generated by different hole inlet edge geometries are obtained using incompressible RANS CFD. These are incorporated into the semi-analytical model, allowing us to determine the effect of changing the hole inlet edge geometry on the hole acoustic response.

This article is structured as follows. After a brief description of the acoustic model in Sec. II we study the sensitivity of the acoustics to the vortex sheet shape in Sec. [II] and the sensitivity of the vortex sheet shape to the hole inlet geometry in Sec. [V] Both analysis are combined in Sec. $\mathrm{V}$ to quantify how different hole inlet shapes affect the hole acoustic responses. Sec. VI presents a parametric study of the effect of the vortex convective velocity on the acoustic response. A summary of results and conclusions are given in Sec.VII.

\section{Problem formulation}

We consider a circular hole of radius $R_{h}$ perforated on a flat plate of thickness $L_{h}$ which separates two half spaces. An axial ( $x_{1}$-direction) uniform flow is imposed upstream which generates a low Mach number "bias flow" passing through the hole. Due to the sudden contraction, the flow separates at the hole inlet edge forming a low-speed recirculation zone close to the orifice edges and a jet through the central part of the hole (Fig. 1]. A shear layer separates these regions.

A small amplitude incoming acoustic wave coming from the upstream side is imposed on this mean flow. Assuming that the Reynolds number is high enough, the perturbed flow field can be considered inviscid everywhere but in the vicinity of the separation point, where viscous effects need to be taken into account to avoid the geometric singularity predicted by inviscid theory. Following Howe [10], we allow vorticity to be shed from the rim of the orifice in such quantities as to eliminate the singularity (via Kutta condition). Subsequently, the vorticity is assumed to be convected 
without change by the mean flow along the shear layer. Any diffusion is neglected.

The stagnation enthalpy $B=C_{p} T+|\mathbf{u}|^{2} / 2$ (where $C_{p}$ denotes the heat capacity at constant pressure, $T$ the temperature and $\mathbf{u}$ the velocity) is used to describe the flow. The flow variables are decomposed into steady mean, denoted by overbar, and perturbation part, denoted by prime. Neglecting volume forces and assuming the flow to be homentropic with constant mean density $\bar{\rho}$ and sound speed $\bar{c}$, the perturbation stagnation entalphy $B^{\prime}$ is governed, as proposed by Howe [28], by

$$
\left(\frac{1}{\bar{c}^{2}}\left(\frac{\partial}{\partial t}+\bar{u} \frac{\partial}{\partial x_{1}}\right)^{2}-\nabla^{2}\right) B^{\prime}=\nabla \cdot\left(\omega^{\prime} \times \boldsymbol{u}_{\boldsymbol{c}}\right)
$$

with boundary conditions

$$
\frac{\partial B^{\prime}}{\partial \mathbf{n}}=0 \quad \text { on the hole side walls. }
$$

Eq. 2 is equivalent to a slip boundary condition for the acoustic perturbation velocity $\mathbf{u}^{\prime} \cdot \mathbf{n}=0$ if we assume vanishing vorticity at the walls. The mean velocity in the acoustic propagation term is assumed to be purely axial ( $x_{1}$-direction) with value $\bar{u}$. The right hand side of Eq. (1) represents the acoustic source term, with $\omega^{\prime}$ the oscillating vorticity [10, 29]. As previously stated, this vorticity is shed at the orifice upstream rim and its strength and phase are determined in accordance with the Kutta condition that the pressure and velocity are finite at that point. In the absence of viscous diffusion, the vorticity will be swept downstream along a infinitely thin sheet (subsequently termed the vortex sheet). In this work, we assume that this vortex sheet corresponds to the streamsurface passing through the upstream hole rim at which separation occurs (see Figs. 1 and 2). The vortex convection velocity is denoted by $\boldsymbol{u}_{\boldsymbol{c}}$ and unless otherwise stated its magnitude is assumed equal to that of the mean velocity at the hole inlet [1, 10, 30, 31]. This assumption is supported by the parametric study of the effect of the vortex convection velocity on the acoustic response provided in Sec. VI

We solve this problem using the semi-analytical approach proposed by Yang and Morgans [19]. A detailed description of the solution method is given in [19, 20, 32]. For the sake of completeness, the main steps of the method are outlined below. First, we introduce a Green's function which is defined in the frequency domain as $\widetilde{G}(\boldsymbol{x}, \boldsymbol{y} ; \omega)$,

$$
\left(\frac{1}{\bar{c}^{2}}\left(-\mathrm{i} \omega+\bar{u} \frac{\partial}{\partial x_{1}}\right)^{2}-\nabla^{2}\right) \widetilde{G}=\delta(\boldsymbol{x}-\boldsymbol{y})
$$

where $\delta$ is the Dirac delta function, $\omega$ is the angular frequency, $\boldsymbol{x}$ denotes the coordinates of the space in which we want to solve the equation and $\boldsymbol{y}$ denotes the acoustic source location in space. Defining a Heaviside function $H(\boldsymbol{x})$ which equals 1 inside the physical domain and 0 outside of it and combining Eqs. (1) and (3), oscillations within the physical 
domain are given by

$$
\widetilde{B}(\boldsymbol{x}, \omega) H=\int_{S}(2 \mathrm{i} k \bar{M} \widetilde{G} \widetilde{B} \boldsymbol{i}+\widetilde{G} \nabla \widetilde{B}-\widetilde{B} \nabla \widetilde{G}) \cdot \mathrm{d} \boldsymbol{s}-\int_{V}\left(\widetilde{\boldsymbol{\omega}} \times \boldsymbol{u}_{\boldsymbol{c}}\right) \cdot \nabla \widetilde{G} \mathrm{~d} v,
$$

where $V$ is the space volume, $S$ denotes the surface bounding the volume, $k=\omega / \bar{c}$ is the acoustic wavenumber, $\mathbf{i}$ is a unit vector in the axial direction and $\mathrm{d} s$ is the area vector in the outwards normal direction to the volume surface.
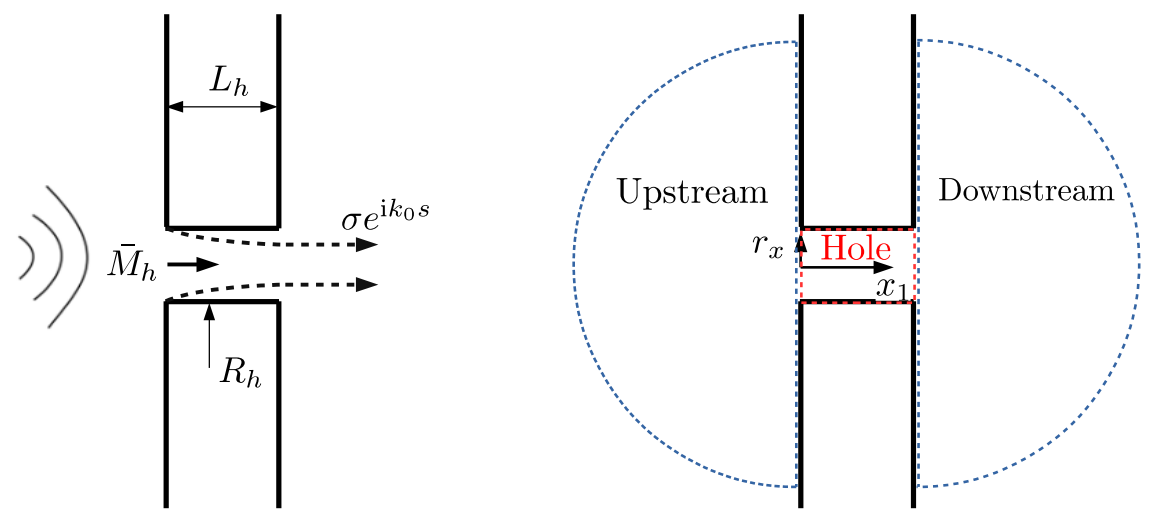

Fig. 2 (Left) A short circular hole opening to half spaces on either side. The unsteady vorticity has strength $\sigma$ and convects with wavenumber $k_{0}=\omega /\left|\mathbf{u}_{\mathbf{c}}\right|$ along the vortex sheet path, $s$. (Right) Upstream, downstream, and hole regions.

As shown in Fig. 2(right), the physical domain is split into three regions : upstream, inside and downstream of the hole. The Green's function which satisfies Eq. (3) can be obtained by incorporating boundary conditions for each region. For the large spaces both up- and downstream, half space Green's functions are used. Fourier Bessel expansions are used to obtain Green's functions inside the hole (see [19] for further details). Since the two half spaces upstream and downstream of the hole have infinite area, the Mach number is zero in those regions. This is not true within the hole, where we assume a finite uniform Mach number.

By substituting the relevant Green's functions into Eq. (4), stagnation enthalpy oscillations in the three regions can be obtained. Velocity oscillations at the hole inlet and outlet are unknown and are expanded as sums of a series of Bessel functions. The curved vortex sheet is discretized along its path into short truncated cone rings. Once full expressions for the stagnation enthalpy oscillations near the hole inlet and outlet have been obtained, the oscillating hole inlet and outlet velocities follow by applying stagnation enthalpy continuity across the hole inlet and outlet interfaces. Stagnation enthalpy oscillations in the up- and downstream regions can then be obtained as a linear function of only the incident plane wave far upstream.

Considering the plane wave oscillations ahead and downstream of the hole, the Rayleigh conductivity of the hole, which denotes the relation between the volume flux oscillation through the hole, $\widetilde{Q}$, and the acoustic pressure oscillation difference across it, $\Delta \widetilde{p}$, is finally obtained through 


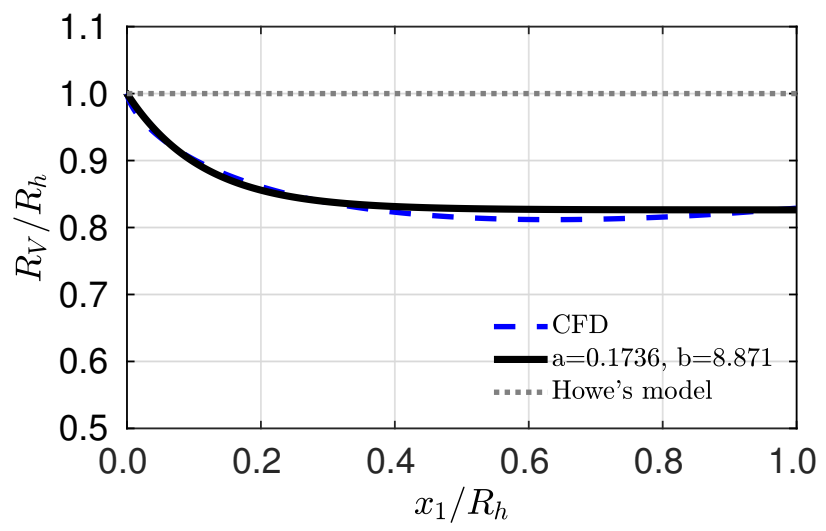

Fig. 3 Vortex sheet shapes: (blue dashed line) extracted from a CFD simulation for a straight-edge hole, (black solid line) fitted by Eq. (7), and (gray dotted line) assumed by Howe's model [10]. $L_{h} / R_{h}=1.0$.

$$
K_{R}=-\frac{\mathrm{i} \omega \bar{\rho} \widetilde{Q}}{\Delta \widetilde{p}}=2 R_{h}\left(\Gamma_{R}-\mathrm{i} \Delta_{R}\right)
$$

$\Gamma_{R}$ represents the inertia of the hole flow and is called the hole reactance. When the hole Rayleigh conductivity is included into a Helmholtz resonator model, accurate knowledge of this reactance is important for accurately predicting the resonator's resonant frequency [2, 6, 20, 33]. $\Delta_{R}$, termed the resistance, is responsible for the absorption $\left(\Delta_{R}\right.$ positive) or generation ( $\Delta_{R}$ negative) of acoustic energy. This is because the acoustic energy absorbed by the hole $\Pi$ can be calculated through $\Pi=\left\langle\operatorname{Re}\left(\Delta \widetilde{p} e^{-\mathrm{i} \omega t}\right) \operatorname{Re}\left(\widetilde{Q} e^{-\mathrm{i} \omega t}\right)\right\rangle$, where Re denotes real part and the angle brackets time average. It then follows that the absorbed acoustic energy,

$$
\Pi=\frac{1}{4}\left(\Delta \widetilde{p}^{*} \widetilde{Q}+\widetilde{Q}^{*} \Delta \widetilde{p}\right)=\frac{R_{h} \Delta_{R}|\Delta \widetilde{p}|^{2}}{\omega \bar{\rho}}
$$

(where [ $]^{*}$ denotes the complex conjugate) is determined by the imaginary part of the Rayleigh conductivity, i.e. the hole resistance.

\section{Effect of the vortex sheet shape on the acoustic response}

A key input parameter of the model presented in Sec. II is the vortex sheet shape. This is the infinitely thin path along which the vorticity shed at the hole inlet edge advects. In this section, we study the effect of this shape on the Rayleigh conductivity of the hole. To this end, we introduce a parametrization for the shape of the vortex sheet using an exponential function of the form

$$
\hat{y}=1-a\left(1-e^{-b \hat{x}}\right)
$$

where $\hat{y}=R_{V} / R_{h}$ and $\hat{x}=x_{1} / R_{h}$ are the normalized radial and axial locations, respectively, of the vortex sheet shape in a cylindrical coordinate system centered at the upstream centre of the hole (see Fig. 2(right)). This parametrization 

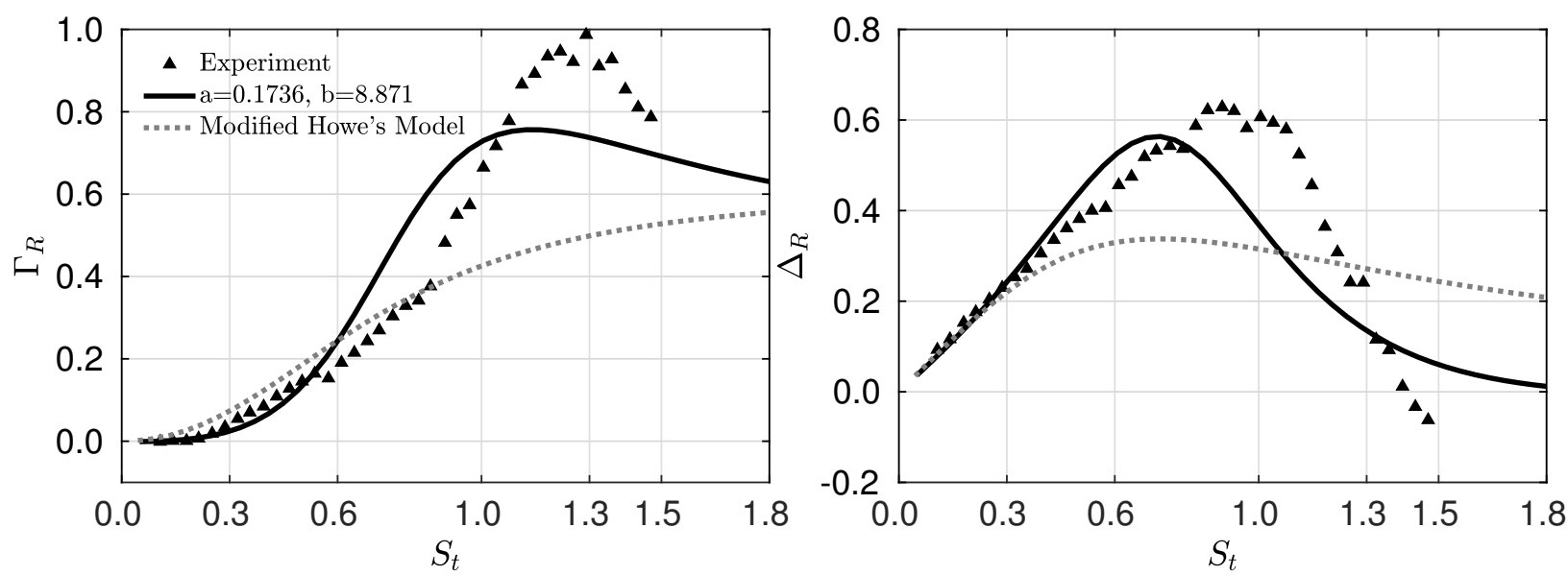

Fig. 4 Rayleigh conductivity: (black triangles) experimental results [17], (black solid line) predicted by the present model, and (gray dotted line) predicted by the modified Howe's model[10, 12]. $L_{h} / R_{h}=1$.

will prove convenient since it describes accurately enough the vortex sheet shapes obtained from simulations using only two parameters: $a$ and $b$. As $\hat{x}$ increases, the radial component becomes $\hat{y} \approx(1-a)$ which approximates the radius of the minimum cross section of the jet (vena contracta). The product $a b$ determines the steepness of the jet at the detachment point $d \hat{y} / d \hat{x}(\hat{x}=0)$. Large values of $a$ correspond to large contractions of the jet profiles. Large values of $b$ correspond to jet profiles contracting sharply after the separation point.

Fig. 3 shows a vortex sheet shape - defined as the streamline passing through the upstream edge of the hole extracted from a RANS simulation for a short hole defined by a length-to-radius ratio $L_{h} / R_{h}=1$ (further details about the simulation are given in Sec. IV). This vortex sheet shape has been fitted using the parametrization given by Eq. (7) to obtain $a=0.1736$ and $b=8.871$. Figure 4 shows the real and imaginary parts of the Rayleigh conductivity predicted by the model using the fitted shape.

In order to assess the quality of the model, the predictions are compared at different dimensionless frequencies $S_{t}=\omega R_{h} /\left|\bar{u}_{h}\right|$ with experimental data from Su et al. [17] and with predictions from the modified Howe's model [10] using a compact hole mass inertial correction [12]. The experimental investigation was undertaken at atmospheric conditions in a test rig consisting of a $120 \mathrm{~mm} \times 120 \mathrm{~mm}$ square cross- sectioned duct which was terminated at each end by large plenum chambers. At one end of the duct a plate with holes was attached. The separation between holes was enough to assure that each hole could be assumed to act in isolation from its neighbour. Attached to the duct were 2 loudspeakers that generated plane acoustic waves which were varied in frequency from $50 \mathrm{~Hz}$ to $1 \mathrm{kHz}$. All the measurements were carried out within the linear absorption range. For the case of relevance here $\left(L_{h} / R_{h}=1.0\right)$, the experimental Mach and Reynolds number based on the mean velocity of the orifice are $M_{h}=0.06$ and $R e=1.7 \times 10^{4}$ respectively (the equivalent values are fed to the model). Figure 4 shows that at very low frequencies $\left(S_{t}<0.4\right)$, both the modified Howe's model (which does not account for details in the vortex sheet shapes) and the present model capture the 
Rayleigh conductivity well, especially $\Delta_{R}$ which is responsible for the acoustic absorption. For $\Gamma_{R}$, it should be noted that experimental errors become significant for very small $\Gamma_{R}$ as the acoustic impedance is dominated by resistance $\left(\Delta_{R}\right)$ at very low frequencies. The experimental $\Gamma_{R}$ and $\Delta_{R}$ start to exhibit much larger values than those predicted by the modified Howe's model for frequencies larger than $S_{t}=0.8$ and $S_{t}=0.4$ respectively. As the frequency further increases beyond $S_{t}=1.0$, the experimental $\Gamma_{R}$ decreases slightly beyond $S_{t}>1.2$ while $\Delta_{R}$ decreases dramatically beyond $S_{t}>1.1$ - even to negative values when $S_{t}>1.4$ which means the hole is generating rather than absorbing acoustics. Both of these important trends are captured by the present model, but not by the modified Howe's model.

It is widely known that the vortex sheet shape is rather sensitive to the hole inlet edge sharpness [17, 22] and, therefore, small variations to the shape considered are expected. To illustrate the effect of vortex sheet shape deviations on the model predictions, we have computed the acoustics response for two additional vortex sheet shapes in Fig. 5 and Fig. $6 \Gamma_{R}$ and $\Delta_{R}$ exhibit a strong sensitivity to the shape of the vortex sheet: $\Gamma_{R}$ is sensitive over the whole considered $S_{t}$ range, while $\Delta_{R}$ shows largest variations in the frequency range $0.6<S_{t}<1.5$.

Experimental results agree better with acoustics predictions for the parametric representation of Eq. (7) given by $a=0.1500, b=6.000$ than with the shape fitted from the simulations for a straight-edge hole. These results suggest that this shape might be closer to the actual experimental one, which is unknown, than the one extracted from simulations. The discharge coefficient $C_{d}$ for the experimental measurements (defined as the actual flow rate/theoretical flow rate through the hole area) further supports this idea. Su et al. [17] obtained an experimental discharge coefficient of 0.73. This is higher than the values reported by Lichtarowickz et al. [34] ranging from 0.63 to 0.67 for the same length-to-radius ratio. Neglecting any friction losses and assuming that the holes are thin enough, the discharge coefficients for the vortex sheet shapes depicted in Fig. 5 can be approximated by the contraction coefficient $\alpha$ (the area of the jet at the vena contracta/area of the hole) to obtain 0.68 and 0.72 for the shapes $a=0.1736, b=8.871$ (fitted from CFD) and $a=0.1500, b=6.000$ respectively. While the discharge coefficient for the shape fitted from the CFD simulation is consistent with the values obtained by Lichtarowickz et al. [34], the discharge coefficient of the vortex sheet shape defined by $a=0.1500, b=6.000$ is closer to the one reported by Su et al. [17]. A certain amount of bluntness of the hole edge in the experiment could explain the higher discharge coefficient and thus the better match with the acoustic measurements for the latter shape.

The model predicts acoustic generation at frequencies beyond $S_{t}=1.7$ and $S_{t}=2$ for the shapes defined by $a=0.1736, b=8.871$ and $a=0.1500, b=6.000$ respectively. This agrees approximately with the "whistling region" (about $1.3<S_{t}<2.5$ ) reported in the literature [35-37]. The predictions of the model for the "whistling region" are greatly affected by uncertainties of the vortex sheet shape and the vortex convective velocity (see Sec.VI).

Figs. 7 and 8 depict a systematic study of the influence of the vortex sheet shape on the acoustic absorption $\Delta_{R}$ at four different frequencies and across two different hole length-to-radius ratios. The acoustic absorption does not exhibit any local extrema in the 2D-planes, but a clear peak is obtained when fixing one of the two parameters. Generally, 


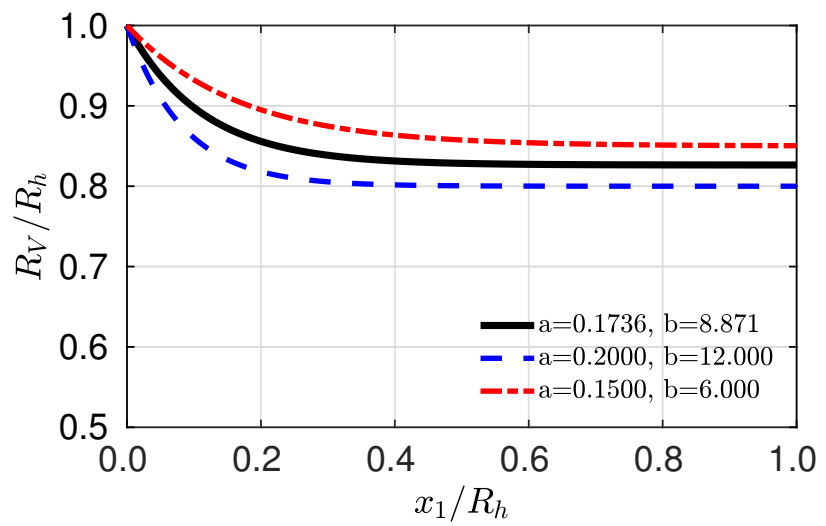

Fig. 5 Various vortex sheet shapes parametrized by Eq. (7) for different values of $a$ and $b$.
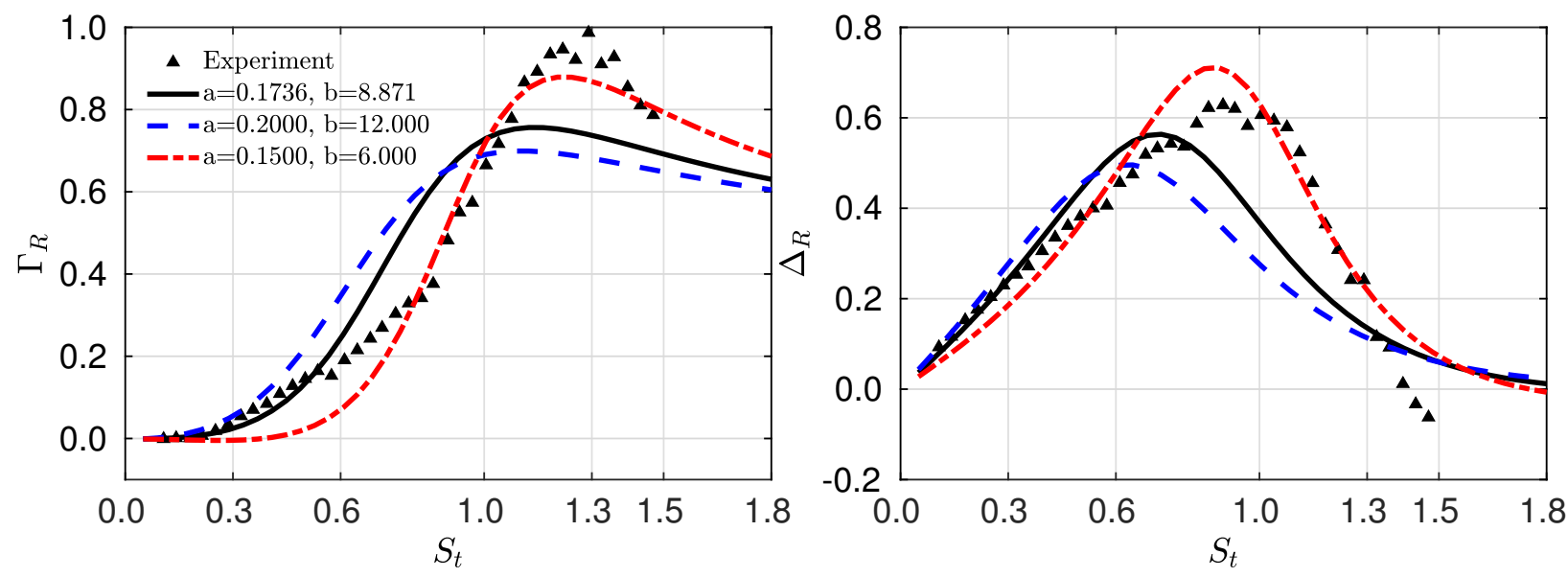

Fig. 6 Rayleigh conductivity predicted by the model using the vortex sheet shapes plotted in Fig. 5 for a hole with $L_{h} / R_{h}=1.0$. (Black triangles) experimental results [17]. 

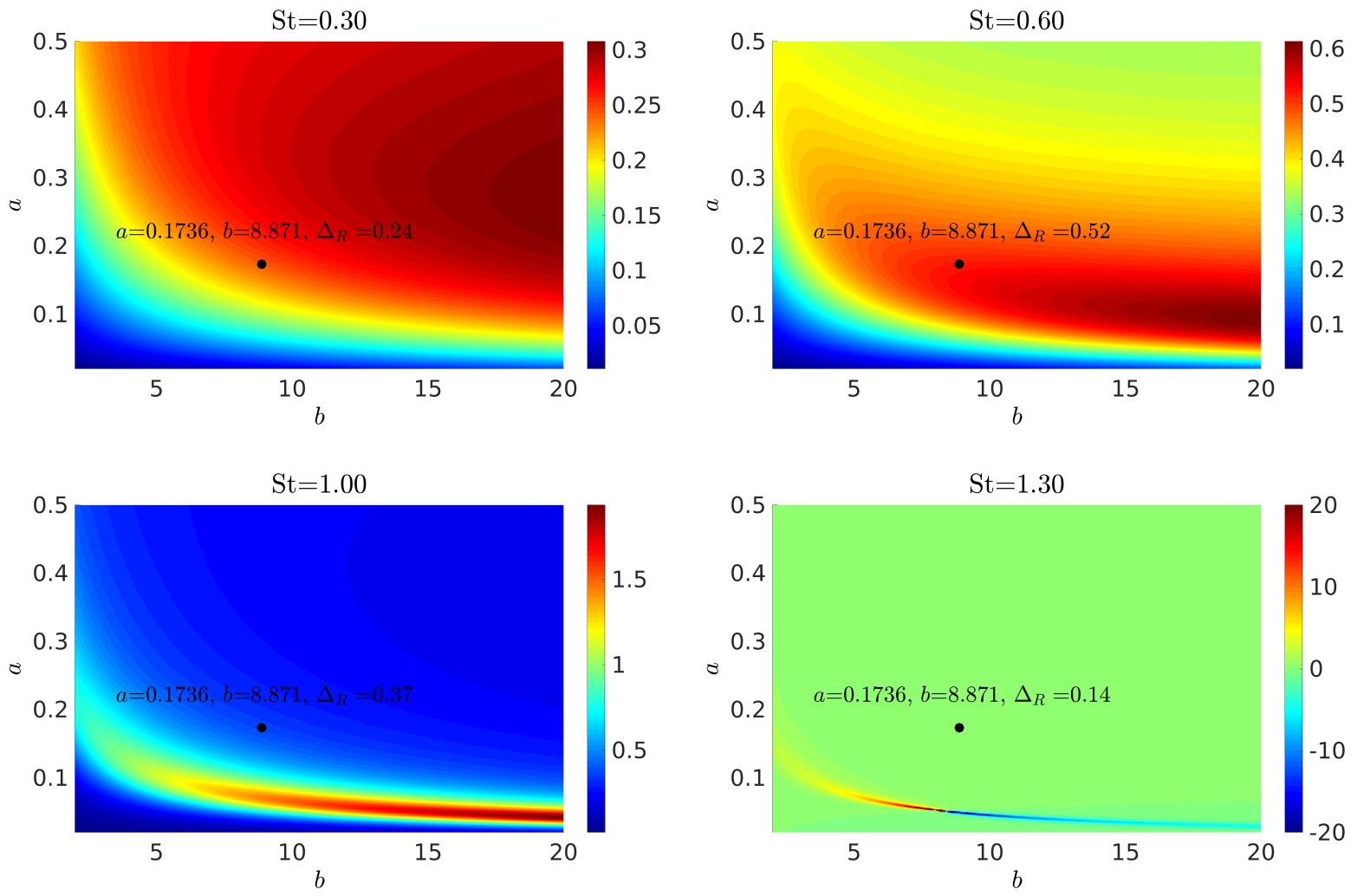

Fig. 7 Imaginary part of the Rayleigh conductivity $\Delta_{R}$ at frequencies: (top left) $S t=0.3$, (top right) $S t=0.6$, (bottom left) $S t=1.0$, and (bottom right) $S t=1.3 . L_{h} / R_{h}=1.0$.

a larger variation of $\Delta_{R}$ is observed at higher $S_{t}$, meaning that the acoustic response is more sensitive to the vortex sheet shape variations at higher frequencies in the considered region. This result has two main implications: (i) the acoustic response of holes can be greatly improved/penalized by small modifications of the vortex sheet shape at higher frequencies, and (ii) at lower frequencies the model predictions for $\Delta_{R}$ are less sensitive to uncertainties in the vortex sheet shape and, therefore, the mismatch with experimental data is lower.

The direction that we need to follow in order to locally maximize the acoustic absorption by modifying a given vortex sheet shape is determined by the direction with the largest gradient of $\Delta_{R}$ in the plane $a-b$. It is clear from Figs. 7 and 8 that this direction is strongly dependent on the frequency of interest.

It is also worth mentioning that negative $\Delta_{R}$ is seen at $S_{t}=1.3$ for the longer hole considered in Fig. 7 but not for the shorter hole considered in Fig. 8. As consistent with previous studies [19, 20, 31, 36, 37] the longer hole is more likely to generate acoustics than the shorter one.

\section{Effect of the hole inlet edge geometry on the vortex sheet}

It is clear that the hole acoustic response exhibits a strong sensitivity to the vortex sheet shape. In this section, we perform the first investigation into how different vortex sheet shapes can be obtained by making very slight modifications 

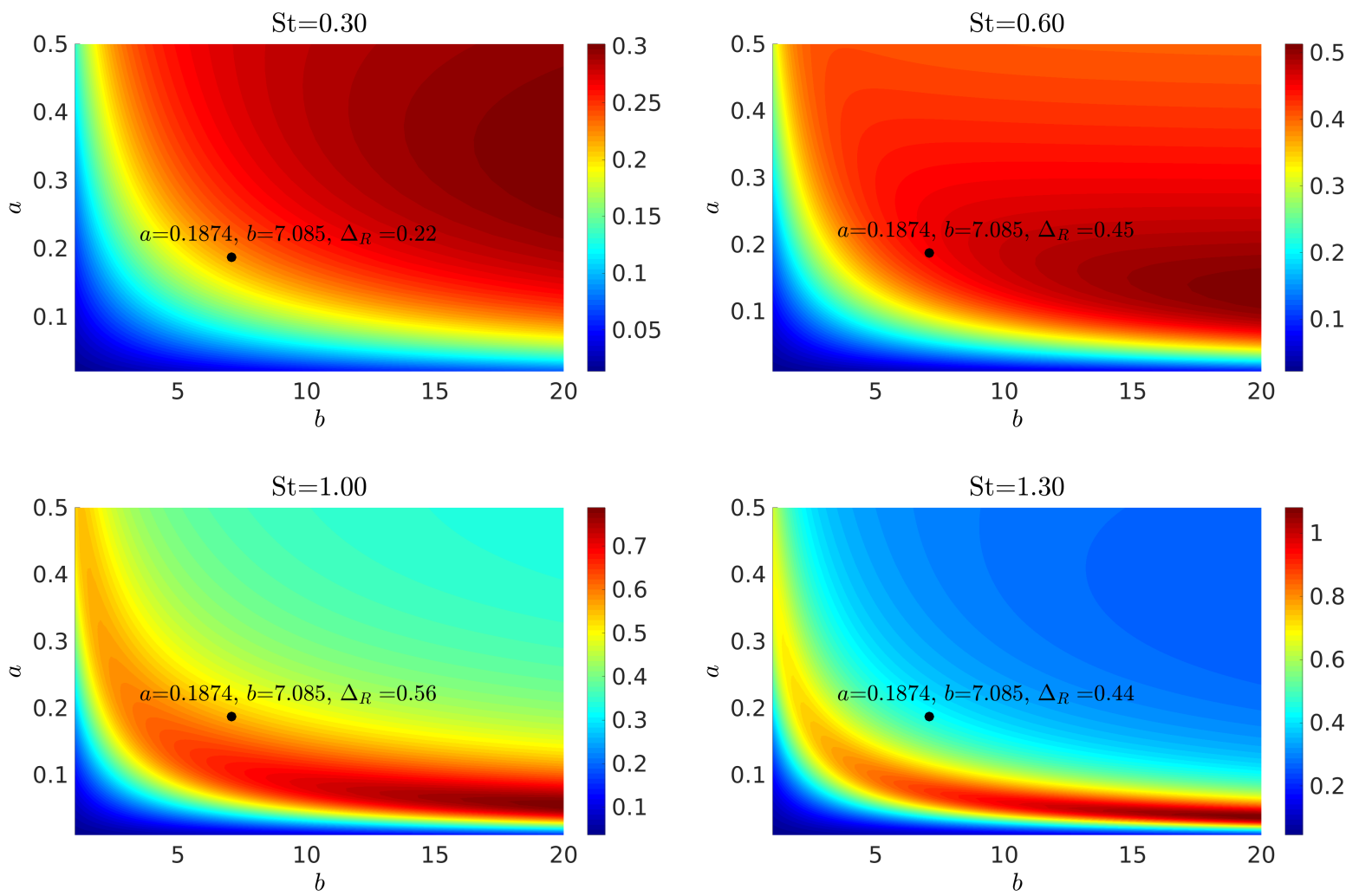

Fig. 8 Imaginary part of the Rayleigh conductivity $\Delta_{R}$ at frequencies: (top left) $S t=0.3$, (top right) $S t=0.6$, (bottom left) $S t=1.0$, and (bottom right) $S t=1.3 . L_{h} / R_{h}=0.5$. 
to the hole inlet edge. To this end, we use RANS simulations to obtain the mean vortex sheet profile for different hole inlet edge geometries. This approach, where the mean flow is studied independently of the acoustics, assumes that acoustic perturbations have a negligible effect on the mean flow near the hole [17].

The computational domain used for the RANS simulations is composed of three sequential concentric cylinders. The first one is placed in the left part of the domain with the flow inlet corresponding to its left base and its length being $200 \mathrm{~mm}\left(40 R_{h}\right)$ and radius $R_{u}$. To its right there is a shorter cylinder corresponding to the hole of interest and whose radius measures $5 \mathrm{~mm}$. Downstream (to the right) of the hole, there is a large cylindrical plenum of length $200 \mathrm{~mm}\left(40 R_{h}\right)$ and radius $R_{d}=R_{u}$. Both the length of the hole and the radius of the inlet duct $R_{u}$ are varied to obtain different length-to-radius ratios $L_{h} / R_{h}$ and hole opening expansion ratios $\lambda=R_{u} / R_{h}$, respectively. Due to the axisymmetric nature of the problem and boundary conditions, the flow is solved in a wedge domain of angle 5 degrees with corresponding boundary conditions in the azimuthal direction. Additional details about the mesh are provided in the Appendix.

To calculate the mean flow, the incompressible RANS equations are converged to a steady state using the finite volume solver OpenFOAM (Version 4.1) [38]. The transport properties are those standard for air at room temperature, i.e. $v=1.5 \times 10^{-5} \mathrm{~m}^{2} / \mathrm{s}$. The inlet normal velocity is varied to obtain three Reynolds numbers: $R e=2 R_{h} \bar{u} / v=10^{4}$, $2 \times 10^{4}$ and $4 \times 10^{4}$. The adopted turbulence model is the Menter $k-\omega$ shear-stress-transport (SST) model due to its performance for free-shear and wall-bounded flows [39]. A SIMPLE algorithm is used to solve the momentum and pressure correction equations. A non-slip boundary condition is imposed on the walls of the hole and downstream plenum. A symmetry condition is imposed on the external walls of the inlet duct.

We first investigate how different geometric/physical parameters affect the vortex sheet shape of the nonmodified/straight-edge hole. Fig. 1 shows an example of a velocity field obtained for an unmodified straight (inlet edge) hole. The theoretical vortex sheet shape can be approximated by the surface of largest azimuthal vorticity [14] or the streamsurface passing through the flow separation line - the upstream rim of the hole in this case. In this paper, we use the latter for two main reasons: (i) assuming the vorticity to be a passive scalar and neglecting any diffusion, it will be convected along that streamsurface by definition, and (ii) it can be accurately and easily extracted in practice.

We perform RANS simulations with the opening confinements upstream and downstream of the hole varying across radius expansion ratios from $\lambda=5$ to $20\left(L_{h} / R_{h}=0.5\right.$ and $R e=2 \times 10^{4}$ are fixed). Results in Fig. 9 (top left) show that the expansion ratio does not noticeably affect the vortex sheet shape when it is larger than $\sim 5$, agreeing with experimental results [40]. However, Fig. 9 (top right), with $\lambda=5$ and $R_{e}=2 \times 10^{4}$, clearly shows that varying the hole length between $0.5 \leq L_{h} / R_{h} \leq 2$ can significantly change the vortex sheet shape. In the following, we consider only the $L_{h} / R_{h}=0.5$ case. This gives a nearly straight vortex sheet tail for $x_{1} / R_{h} \geq 0.5$ and thus allows us to use the two parameter expression in Eq. (7) for modeling. Note that more complex vortex sheet shapes could be considered by the present semi-analytical model - the only requirement is that the hole is short enough to avoid mean flow reattachment 

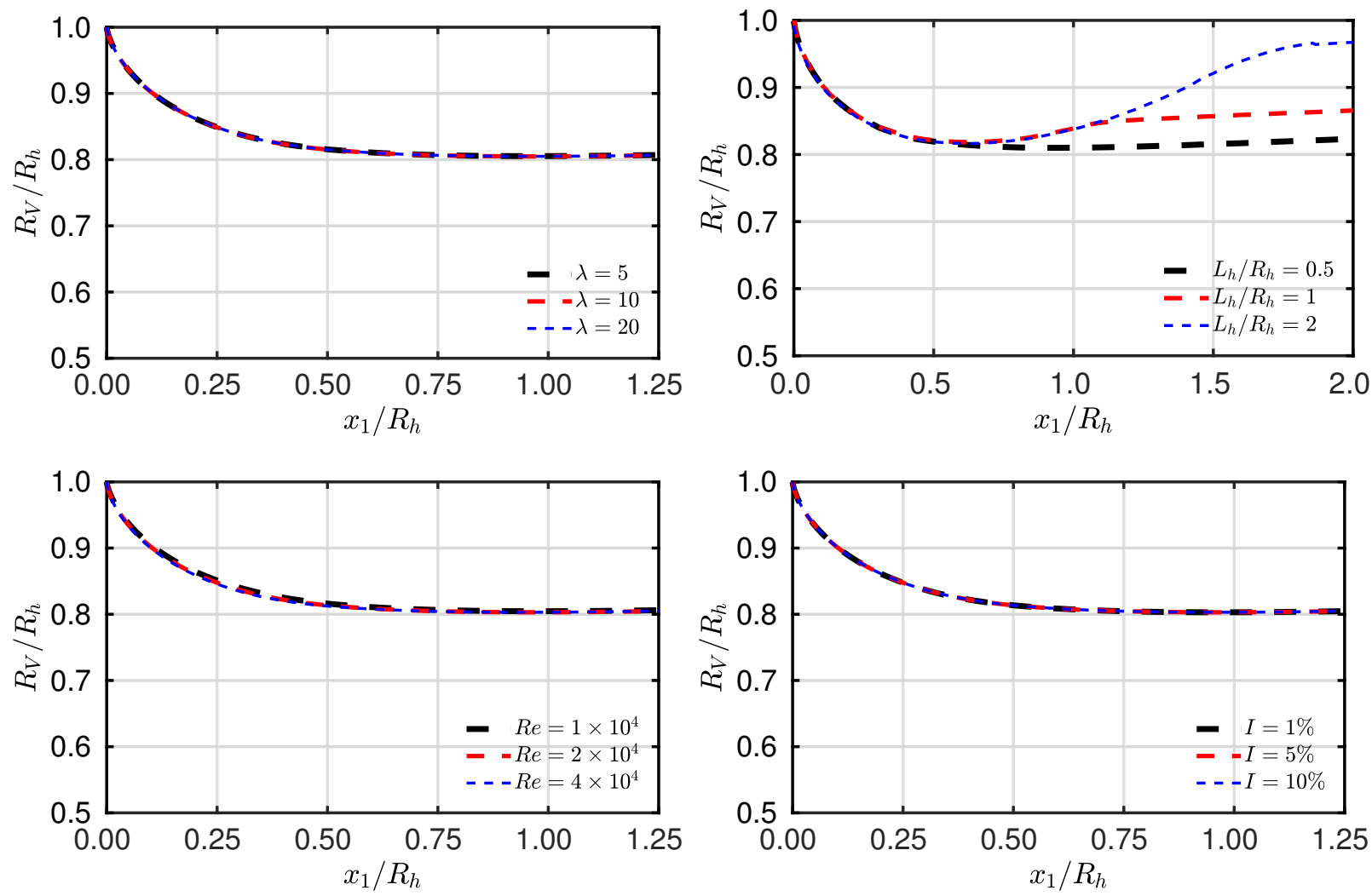

Fig. 9 Influence on the vortex sheet shape of : (top left) expansion ratio $\lambda$, (top right) length-to-radius ratio $L_{h} / R_{h}$, (bottom left) Reynolds number $R e$, and (bottom right) turbulent intensity $I$. 
within it [19, 20].

In Fig. 9 (bottom left), we show the results for different Reynolds numbers $R e$ with $\lambda$ fixed to 5 and $L_{h} / R_{h}=0.5$. At these high Reynolds numbers (common in acoustic dampers for aero-engine and power gas turbines), the vortex sheet shape is insensitive to variations of the Reynolds number [34]. Figure 9 (bottom right) shows that the turbulence intensity $I$ at the inlet of the domain $\left(\lambda=5, L_{h} / R_{h}=0.5\right.$, and $\left.R e=2 \times 10^{4}\right)$ does not impact the vortex sheet shape. The last two results indicate that the shape of the mean flow jet profile inside the hole is determined by inertial terms and, therefore, by the geometry of the lip of the hole.
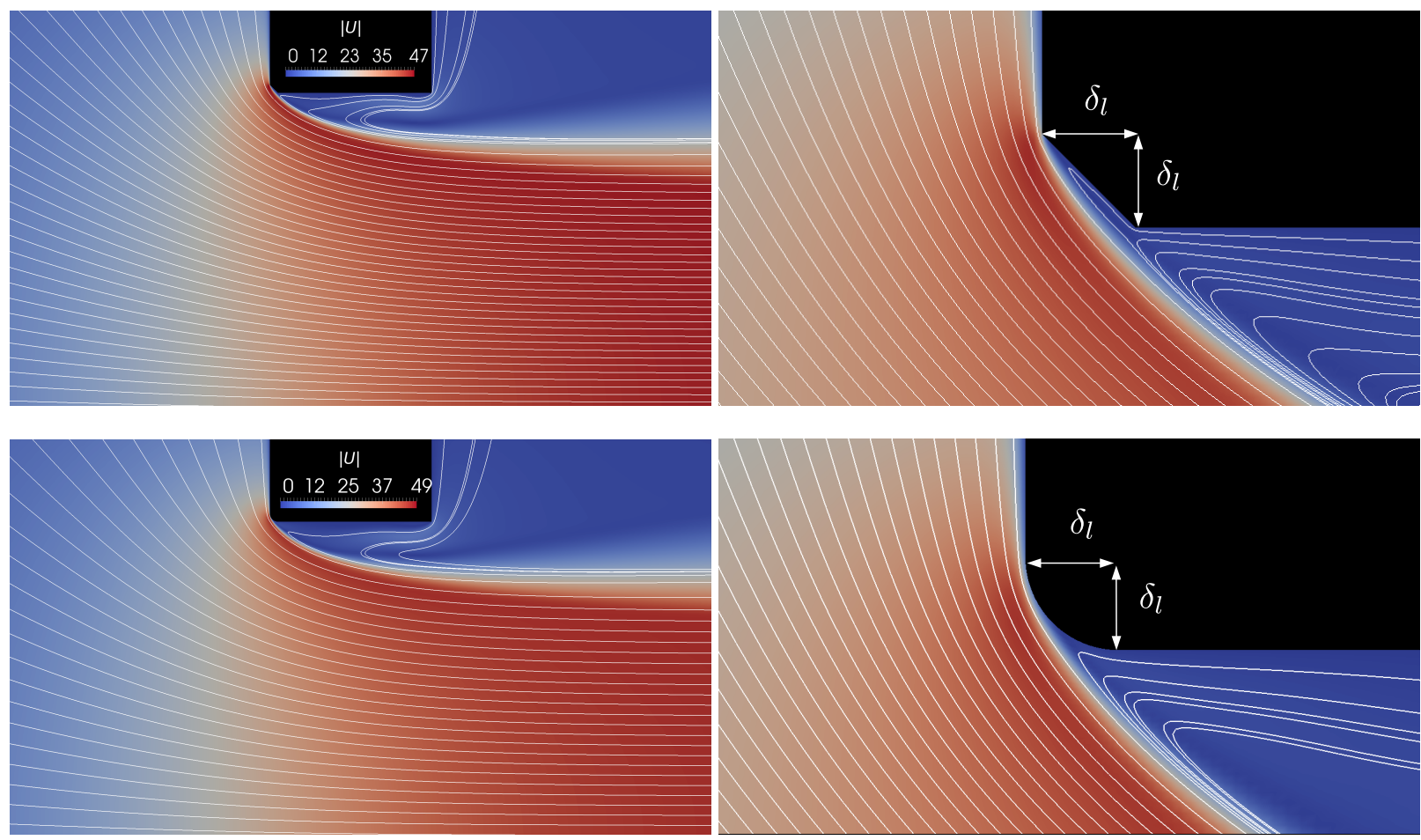

Fig. 10 Mean flow velocity magnitude $|\overline{\mathbf{u}}|$ for holes with : (top) a chamfered-edge and (bottom) a rounded-edge. The geometry is defined by $L_{h} / R_{h}=0.5$ and $\delta_{l} / R_{h}=2.5 \%$.

Hereafter, we fix $\lambda=5, R e=2 \times 10^{4}, I=1 \%$ and $L_{h} / R_{h}=0.5$ and we study cases with modified inlet edges. We consider four geometries: (i) a chamfered edge, (ii) a rounded edge, (iii) an elliptical edge with the major axis placed horizontally, and (iv) an elliptical edge with the major axis placed vertically. Fig. 10 shows the chamfered and rounded edge cases with the modification $\delta_{l} / R_{h}=2.5 \%$ being very small compared to the radius of the hole. The mesh near the edge is carefully refined to make sure that the flow separation is accurately captured. To this end, a sensitivity analysis of the mesh in that region has been systematically performed in the Appendix.

Even though the edge modifications are very small, the extracted vortex sheet shapes in Fig. 11 exhibit noticeable differences compared to the straight case, which confirms high sensitivity to the hole inlet edge shape. The vortex sheet shape from the straight edge case agrees approximately with results from [40]. The chamfered and rounded cases both 


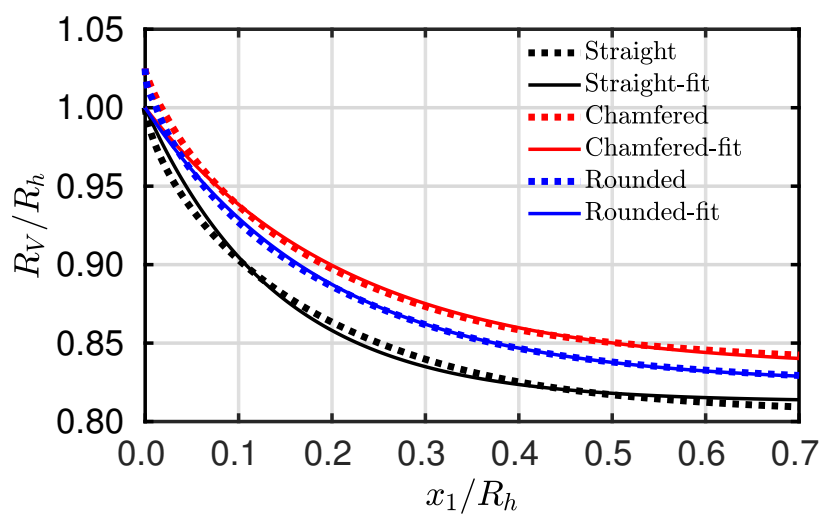

Fig. 11 Vortex sheet shapes for different hole-inlet-edge shapes: (dotted lines) extracted from CFD simulations and (solid lines) fitted by Eq. (7). $L_{h} / R_{h}=0.5$.

give a less contracted shape. Note that the difference between both is small but noticeable with the chamfered one exhibiting a larger contraction.

Equation (7) is used to fit these vortex sheet shapes. The fitted results are plotted in Fig. 11 with $a=0.1874, b=7.085$ for the straight case, $a=0.1663, b=4.632$ for the chamfered case and $a=0.1760, b=5.108$ for the rounded case. It can be seen that the shapes for the chamfered and rounded cases are well captured by the corresponding fitted curves, although with slight differences near the edge.
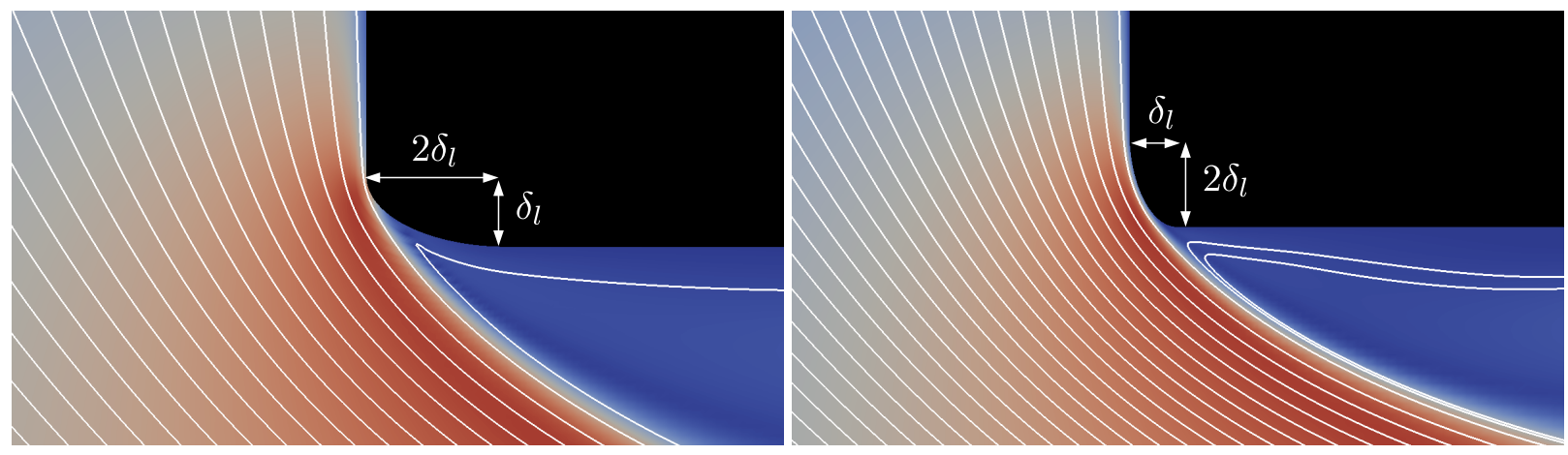

Fig. 12 Zoomed-in view near the inlet edge of the mean flow velocity magnitude $|\overline{\mathbf{u}}|$ for two elliptical-edge holes with $L_{h} / R_{h}=0.5 . \delta_{l} / R_{h}=2.5 \%$.

Fig. 12 shows a zoom-in view of the velocity field near the hole edge for the two elliptical cases. We compare the vortex sheet shape for both cases with the straight and rounded edges in Fig. 13. It can be seem that the shapes for the rounded and the two elliptical cases are rather similar with small differences only noticeable close to the edge. The fitting parameters are $a=0.1710, b=5.090$ and $a=0.1741, b=4.518$ for the ellipse with horizontal and vertical major axis, respectively. These fitting values suggest that the acoustic performance of the two elliptical and the rounded edges might be similar. This analysis however neglects the discrepancies of the three vortex sheet shapes in the vicinity of the hole inlet. For edge modifications of a larger scale than the ones considered in the present study, this region might 


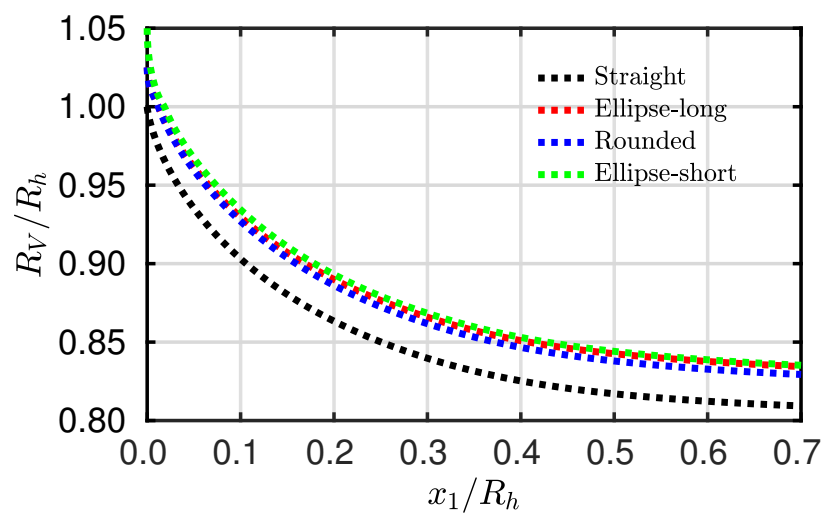

Fig. 13 Vortex sheet shapes extracted from CFD simulations for different hole-inlet-edge shapes. $L_{h} / R_{h}=0.5$.

have an important impact on the acoustics and further studies would be necessary.

\section{Effect of the hole inlet edge geometry on the acoustic response}
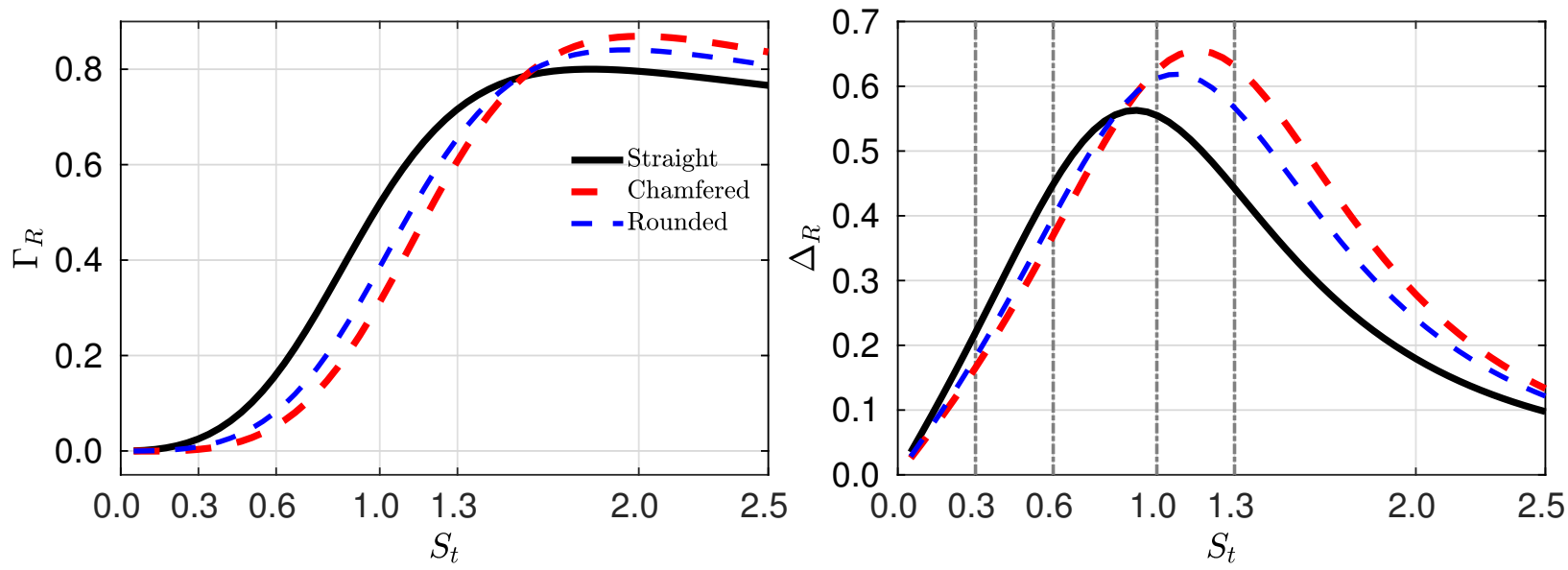

Fig. 14 Rayleigh conductivity predicted by the model for holes with different inlet-edge shapes. $L_{h} / R_{h}=0.5$. Gray dash-dot lines correspond to the frequencies depicted in Fig. 8 .

In this section, we put together the results obtained in Sec. III and Sec. IV to quantify the impact of the geometry on the acoustic response of the hole by incorporating the fitted vortex sheet shapes into the present semi-analytical model.

We compare the straight, chamfered and rounded cases. The Rayleigh conductivities across a wide range of frequencies are shown in Fig. 14. Both the chamfered and rounded holes give significantly different results compared to the straight one even though their edge modification is small. This is especially true for $\Delta_{R}$ in the frequency range $1 \leq S_{t} \leq 2, \Delta_{R}$ for the modified holes can be $30 \% \sim 50 \%$ larger than for the straight hole case, meaning that the sound absorption can be $30 \% \sim 50 \%$ higher. At high frequencies, we found that the chamfered edge hole is the best shape for maximising acoustic absorption, with the straight edge hole exhibiting the lowest absorptions. In contrast, at low frequencies, these shapes invert roles, with the largest absorptions given by the straight edge and the lowest by 
the chamfered-edge hole. In practice, the absorption depends not only on the Rayleigh conductivity, but also on the oscillating pressure difference across a hole (Eq. 6) and, thus, the acoustic boundary conditions either side of it.

The hole reactance, $\Gamma_{R}$, can be much lower than for the straight hole at frequencies below $S_{t}=1.3(10 \% \sim 80 \%$ lower depending on its absolute value at different frequencies).

\section{Effect of the vortex convection velocity on the acoustic response}

Throughout this paper, the vortex convection velocity is assumed constant and equal to the mean velocity at the hole inlet. This assumption was first introduced by Howe [10, 28] and has been used in a number of studies [1, 30, 31] since. To justify it, Howe [10] proposed the vortex convection velocity $\left|\mathbf{u}_{c}\right|=0.5 \bar{u}_{\sigma}$ for an infinitely thin hole (with $\bar{u}_{\sigma}$ the magnitude of the velocity at the vena contracta). Using the conservation of mass through the hole, the mean velocity at the inlet of the hole can be related to this velocity by $\bar{u}_{h}=\alpha \bar{u}_{\sigma}$, with $\alpha$ the contraction coefficient. Assuming that the contraction coefficient is the minimal theoretical value $\alpha=0.5$ [41], the vortex convection velocity can be expressed as $\left|\mathbf{u}_{c}\right|=\bar{u}_{h}$. However, experimental and numerical values reported in the literature range from 0.61 to 0.67 , making the previous expression an approximation.

Fig. 15 depicts the Rayleigh conductivity predicted by the model using three different vortex convection velocities: (a) the mean velocity at the inlet of the hole, (b) the velocity at the vena contracta and (c) the theoretical velocity proposed by Howe [10, 28] (half of the velocity at the contracta). The value of the Rayleigh conductivity at the peak is similar for the three cases, but the frequency at which the peak is located exhibits a strong sensitivity to the vortex convection velocity, with the maximums in $\Delta_{R}$ and $\Gamma_{R}$ shifting to lower frequencies with lower velocities. The vortex convection velocity which predicts the Rayleigh conductivity in closest agreement with the experimental results is the velocity at the inlet of the hole. $\left|\mathbf{u}_{c}\right|=\bar{u}_{\sigma}$ and $\left|\mathbf{u}_{c}\right|=0.5 \bar{u}_{\sigma}$ over- and under-predict the frequency of the peak (and the convective velocity) respectively. This result suggests that an appropriate value for the vortex convection velocity will be in between these two values. The range $0.6-0.8$ times the magnitude of the velocity at the vena contracta might be reasonable choice. The velocity at the inlet of the hole falls into this range and seems a plausible approximation.

\section{Conclusions}

The present paper provides a theoretical and numerical study of the sensitivity of the acoustics of short circular holes to their inlet edge geometries, achieved by slightly modifying the inlet edge shape. The theoretical part relies on a recently developed semi-analytical model which accounts for detailed vortex-sound coupling for short circular holes with mean bias flows. This makes the study of the acoustic response of holes with different vortex sheet shapes very efficient. As the vortex sheet shape is strongly sensitive to the shape of the hole inlet edge, but assumed insensitive to small acoustic perturbations, we use incompressible RANS simulations to extract it. The acoustic response is then obtained by combining these two parts. This approach provides a very efficient tool to study this problem and paves the 

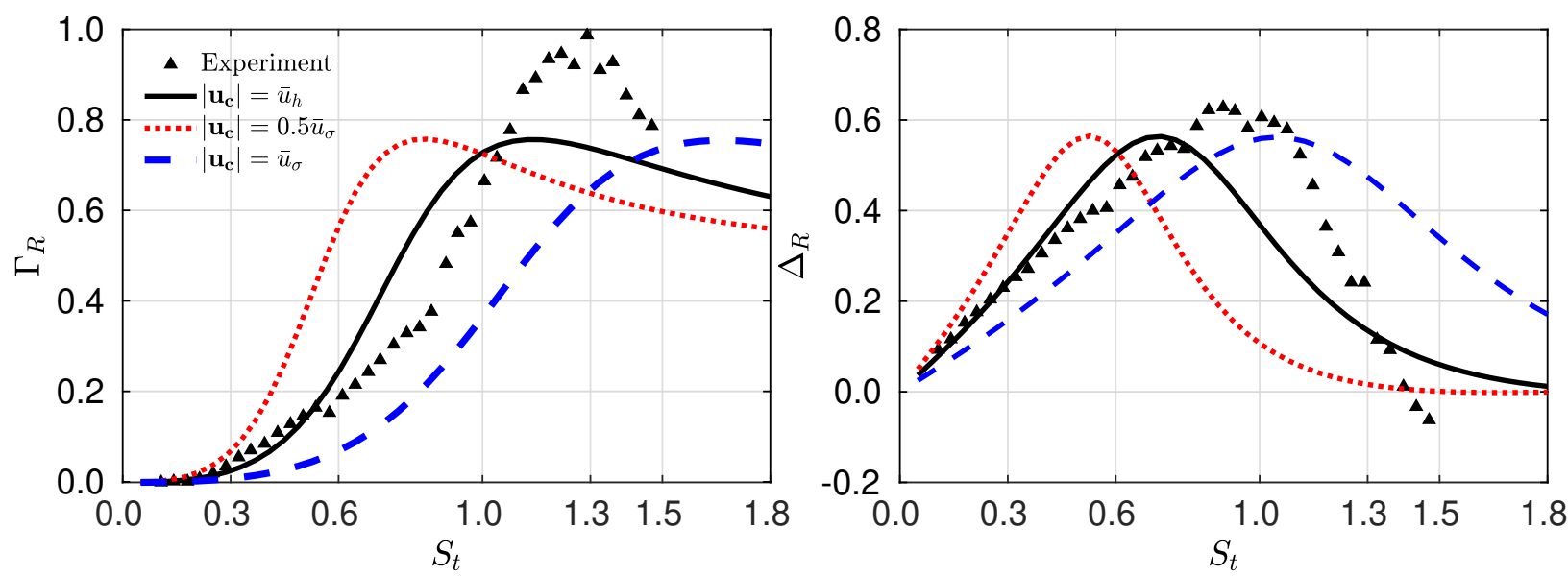

Fig. 15 Rayleigh conductivity predicted by the present model for different vortex convection velocities. (Black triangles) experimental results [17]. $L_{h} / R_{h}=1.0$.

way to systematically optimize the acoustics of short circular holes by modifying their inlet edge shapes.

We have shown that very small changes of the hole inlet edge can bring about significant variations in the vortex sheet shape, and corresponding significant modifications to the hole acoustic response. This is specially true at higher frequencies, where the acoustic absorption presents a stronger sensitivity. Additionally, we have found that rounded/chamfered edges create less contracted mean jet profiles than straight edges. This, in turn, leads to a significant increase in acoustic absorption at high frequencies and a slight decrease at low frequencies as compared to a straight hole. From an application point of view, these results stress the importance of carefully manufacturing short holes with mean bias flows (such as acoustic liners and Helmholtz resonators) in order to ensure good acoustic performance.

The method outlined in this paper is restricted to small modifications of the edge when compared to the radius of the hole. If we aim to account for larger modifications, these must be included into the acoustic analysis. This challenge is currently being explored and will be addressed in a forthcoming study.

\section{Appendix A. Mesh sensitivity analysis}

In this appendix, we study the influence of the mesh density on the vortex sheet shapes. We set the physical and geometric parameters of the RANS simulation at $\lambda=5, L_{h} / R_{h}=0.5, R e=2 \times 10^{4}$ and $I=1 \%$ through this appendix. All the meshes are structured meshes.

Fig. 16 depicts the vortex sheet shape obtained for three different meshes for a straight edge hole. The meshes denoted as coarse, medium and fine are composed of 19890, 52960 and 73185 cells, respectively. As we can observe, the vortex sheet shape is accurately captured by the three meshes.

Fig. 17 shows the vortex sheet shape obtained for the four edge shapes addressed in this paper. The sizes of the coarse and fine meshes respectively are 24588 and 73980 for the chamfered edge, 23500 and 47275 for the rounded edge, 22128 and 48200 for the elliptical with horizontal major axis edge; and 20048 and 47545 for the elliptical with 


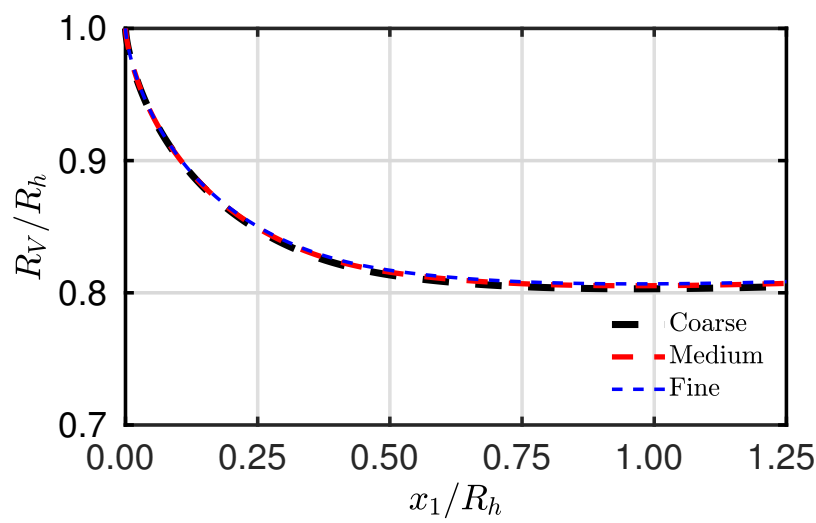

Fig. 16 Influence of the mesh density on the vortex sheet shape for a straight-edge hole.

vertical major axis edge. Again, the vortex sheet shapes are confirmed insensitive to mesh refinement.

\section{Funding Sources}

The authors would like to gratefully acknowledge the European Research Council (ERC) Starting Grant ACOULOMODE (2013-2018), the ERC Consolidator Grant AFIRMATIVE (2018-2023) and the Engineering and Physical Sciences Research Council (EPSRC) Grant CHAMBER (2017-2020) for supporting the current research.

\section{References}

[1] Eldredge, J. D., and Dowling, A. P., “The absorption of axial acoustic waves by a perforated liner with bias flow," Journal of Fluid Mechanics, Vol. 485, 2003, pp. 307-335. doi:10.1017/S0022112003004518.

[2] Dupère, I. D. J., and Dowling, A. P., “The use of Helmholtz resonators in a practical combustor," Journal of Engineering for Gas Turbines and Power, Vol. 127, No. 2, 2005, pp. 268-275. doi:10.1115/1.1806838.

[3] Zhao, D., and Li, X. Y., "A review of acoustic dampers applied to combustion chambers in aerospace industry," Progress in Aerospace Sciences, Vol. 74, 2015, pp. 114-130. doi:10.1016/j.paerosci.2014.12.003.

[4] Ni, F., Miguel-Brebion, M., Nicoud, F., and Poinsot, T., "Accounting for acoustic damping in a Helmholtz solver," AIAA Journal, Vol. 55, No. 4, 2016, pp. 1-16. doi:10.2514/1.J055248.

[5] Yang, D., Wang, X., and Zhu, M., "The impact of the neck material on the sound absorption performance of Helmholtz resonators," Journal of Sound and Vibration, Vol. 333, No. 25, 2014, pp. 6843-6857. doi:10.1016/j.jsv.2014.07.034.

[6] Yang, D., and Morgans, A. S., “Acoustic Models for Cooled Helmholtz Resonators," AIAA Journal, Vol. 55, No. 9, 2017 , pp. 3120-3127. doi:10.2514/1.J055854.

[7] Candel, S., Durox, D., Schuller, T., Bourgouin, J.-F., and Moeck, J. P., "Dynamics of swirling flames," Annual Review of Fluid Mechanics, Vol. 46, 2014, pp. 147-173. doi:10.1146/annurev-fluid-010313-141300. 

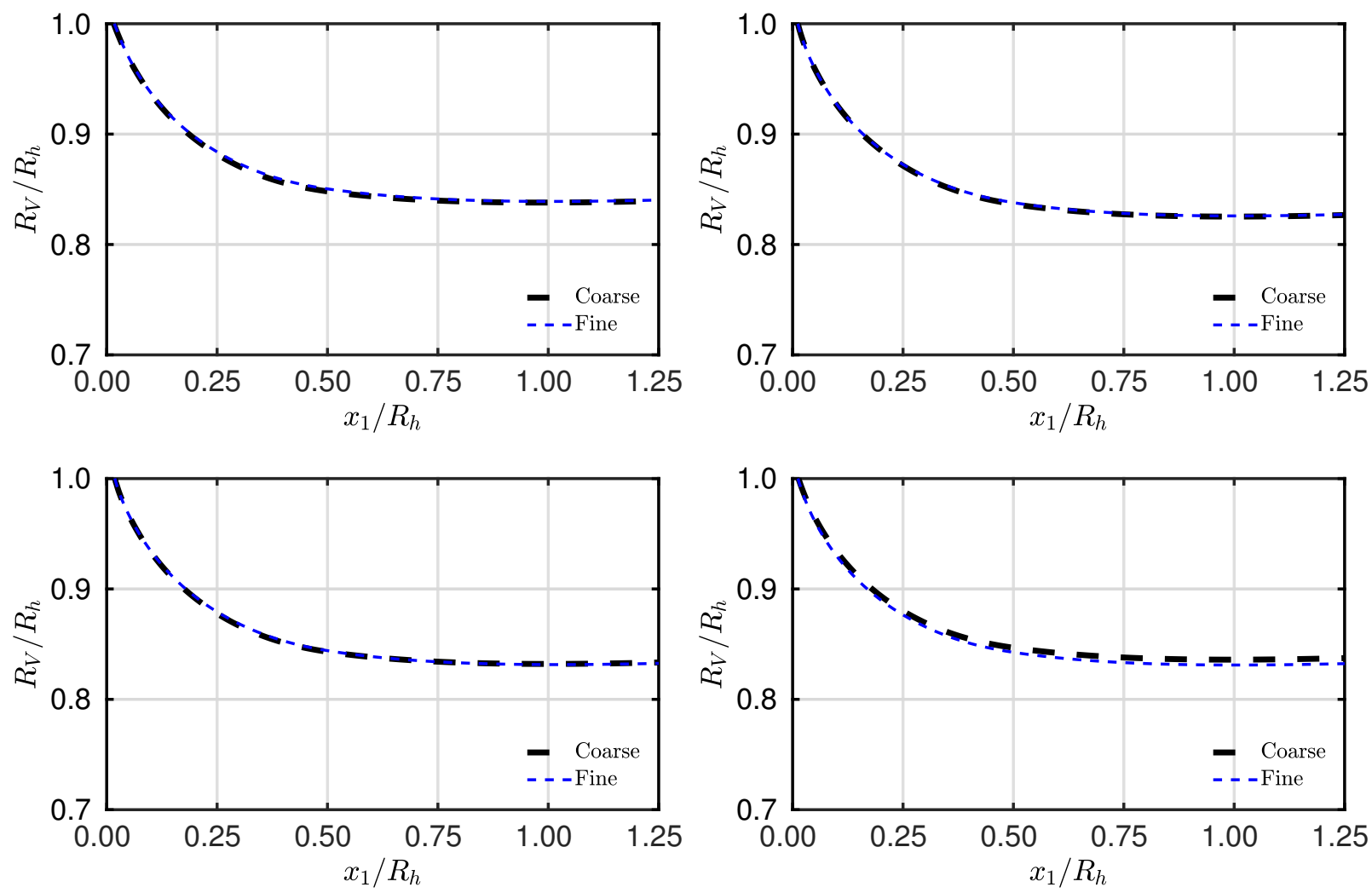

Fig. 17 Mesh sensitivity for holes with different edges : (top left) chamfered, (top right) rounded, (bottom left) elliptical with vertical major axis, and (bottom right) elliptical with horizontal major axis. 
[8] Howe, M. S., and McGowan, R. S., "Production of sound by unsteady throttling of flow into a resonant cavity, with application to voiced speech,” Journal of Fluid Mechanics, Vol. 672, 2011, pp. 428-450. doi:10.1017/S0022112010006117.

[9] Howe, M. S., "Attenuation of sound in a low Mach number nozzle flow," Journal of Fluid Mechanics, Vol. 91, No. 02, 1979, pp. 209-229. doi:10.1017/S0022112079000124.

[10] Howe, M. S., "On the theory of unsteady high Reynolds number flow through a circular aperture," Proceedings of the Royal Society of London. A. Mathematical and Physical Sciences, Vol. 366, No. 1725, 1979, pp. 205-223. doi:10.1098/rspa.1979.0048.

[11] Dowling, A., and Hughes, I., "Sound absorption by a screen with a regular array of slits," Journal of Sound and Vibration, Vol. 156, No. 3, 1992, pp. 387-405. doi:10.1016/0022-460X(92)90735-G.

[12] Jing, X., and Sun, X., "Experimental investigations of perforated liners with bias flow," The Journal of the Acoustical Society of America, Vol. 106, No. 5, 1999, pp. 2436-2441. doi:10.1121/1.428128.

[13] Bellucci, V., Flohr, P., and Paschereit, C. O., "Numerical and Experimental Study of Acoustic Damping Generated by Perforated Screens," AIAA journal, Vol. 42, No. 8, 2004, pp. 1543-1549. doi:10.2514/1.9841.

[14] Mendez, S., and Eldredge, J. D., "Acoustic modeling of perforated plates with bias flow for large-eddy simulations," Journal of Computational Physics, Vol. 228, No. 13, 2009, pp. 4757-4772. doi:10.1016/j.jcp.2009.03.026.

[15] Scarpato, A., Tran, N., Ducruix, S., and Schuller, T., "Modeling the damping properties of perforated screens traversed by a bias flow and backed by a cavity at low Strouhal number," Journal of Sound and Vibration, Vol. 331, No. 2, 2012, pp. $276-290$. doi:10.1016/j.jsv.2011.09.005.

[16] Dai, X., Jing, X., and Sun, X., "Acoustic-excited vortex shedding and acoustic nonlinearity at a rectangular slit with bias flow," Journal of Sound and Vibration, Vol. 333, No. 13, 2014, pp. 2713-2727. doi:10.1016/j.jsv.2014.02.029.

[17] Su, J., Rupp, J., Garmory, A., and Carrotte, J. F., "Measurements and computational fluid dynamics predictions of the acoustic impedance of orifices," Journal of Sound and Vibration, Vol. 352, 2015, pp. 174-191. doi:10.1016/j.jsv.2015.05.009.

[18] Lahiri, C., and Bake, F., "A review of bias flow liners for acoustic damping in gas turbine combustors," Journal of Sound and Vibration, Vol. 400, 2017, pp. 564-605. doi:10.1016/j.jsv.2017.04.005.

[19] Yang, D., and Morgans, A. S., "A semi-analytical model for the acoustic impedance of finite length circular holes with mean flow," Journal of Sound and Vibration, Vol. 384, 2016, pp. 294-311. doi:10.1016/j.jsv.2016.08.006.

[20] Yang, D., and Morgans, A. S., “The acoustics of short circular holes opening to confined and unconfined spaces," Journal of Sound and Vibration, Vol. 393, 2017, pp. 41-61. doi:10.1016/j.jsv.2016.12.027.

[21] Chen, Z., Ji, Z., and Huang, H., “Acoustic impedance of perforated plates in the presence of bias flow," Journal of Sound and Vibration, Vol. 446, 2019, pp. 159-175. doi:10.1016/j.jsv.2019.01.031. 
[22] Bullen, P. R., Cheeseman, D. J., and Hussain, L. A., "The effects of inlet sharpness on the pipe contraction pressure loss coefficient," International Journal of Heat and Fluid Flow, Vol. 9, No. 4, 1988, pp. 431-433. doi:10.1016/0142-727X(88)90012-4.

[23] Keller, J. J., and Zauner, E., "On the use of Helmholtz resonators as sound attenuators," Zeitschrift für angewandte Mathematik und Physik ZAMP, Vol. 46, No. 3, 1995, pp. 297-327. doi:10.1007/BF01003552.

[24] Rupp, J., "Acoustic absorption and the unsteady flow associated with circular apertures in a gas turbine environment,” Ph.D. thesis, 2013. doi:2134/12984.

[25] Lahiri, C., "Acoustic performance of bias flow liners in gas turbine combustors," Ph.D. thesis, 2014. doi:10.14279/depositonce4270.

[26] Ji, C., Zhao, D., Han, N., and Li, J., "Parametric measurements of the effect of in-duct orifice edge shape on its noise damping performance," Journal of Sound and Vibration, Vol. 384, 2016, pp. 130-145. doi:10.1016/j.jsv.2016.08.007.

[27] Ji, C., and Zhao, D., "Two-dimensional lattice Boltzmann investigation of sound absorption of perforated orifices with different geometric shapes," Aerospace Science and Technology, Vol. 39, 2014, pp. 40-47. doi:10.1016/j.ast.2014.08.010.

[28] Howe, M. S., Acoustics of Fluid-Structure Interactions, Cambridge University Press, Cambridge, United Kingdom, 1998. doi:10.1017/CBO9780511662898.

[29] Dupère, I. D. J., and Dowling, A. P., “The absorption of sound near abrupt axisymmetric area expansions,” Journal of Sound and Vibration, Vol. 239, No. 4, 2001, pp. 709-730. doi:10.1006/jsvi.2000.3224.

[30] Hughes, I. J., and Dowling, A. P., “The absorption of sound by perforated linings,” Journal of Fluid Mechanics, Vol. 218, 1990, pp. 299-335. doi:10.1017/S002211209000101X.

[31] Jing, X., and Sun, X., "Effect of plate thickness on impedance of perforated plates with bias flow," AIAA Journal, Vol. 38, No. 9, 2000, pp. 1573-1578. doi:10.2514/2.1139.

[32] Yang, D., “The acoustics of short circular holes and their damping of thermoacoustic oscillations,” Ph.D. thesis, 2017.

[33] Bellucci, V., Flohr, P., Paschereit, C. O., and Magni, F., "On the use of Helmholtz resonators for damping acoustic pulsations in industrial gas turbines," Journal of Engineering for Gas Turbines and Power, Vol. 126, No. 2, 2004, pp. $271-275$. doi:10.1115/1.1473152.

[34] Lichtarowicz, A., Duggins, R., and Markland, E., "Discharge coefficients for incompressible non-cavitating flow through long orifices," Journal of mechanical engineering science, Vol. 7, No. 2, 1965, pp. 210-219. doi:10.1243/JMES_JOUR_1965_007_ 029_02.

[35] Testud, P., Aurégan, Y., Moussou, P., and Hirschberg, A., "The whistling potentiality of an orifice in a confined flow using an energetic criterion," Journal of Sound and Vibration, Vol. 325, No. 4, 2009, pp. 769-780. doi:10.1016/j.jsv.2009.03.046. 
[36] Lacombe, R., Moussou, P., and Aurégan, Y., "Whistling of an orifice in a reverberating duct at low Mach number," The Journal of the Acoustical Society of America, Vol. 130, No. 5, 2011, pp. 2662-2672. doi:10.1121/1.3641427.

[37] Kierkegaard, A., Allam, S., Efraimsson, G., and Åbom, M., "Simulations of whistling and the whistling potentiality of an in-duct orifice with linear aeroacoustics," Journal of Sound and Vibration, Vol. 331, No. 5, 2012, pp. $1084-1096$. doi:10.1016/j.jsv.2011.10.028.

[38] Weller, H. G., Tabor, G., Jasak, H., and Fureby, C., "A tensorial approach to computational continuum mechanics using object-oriented techniques," Computers in physics, Vol. 12, No. 6, 1998, pp. 620-631. doi:10.1063/1.168744.

[39] Menter, F. R., Kuntz, M., and Langtry, R., “Ten years of industrial experience with the SST turbulence model,” Turbulence, heat and mass transfer, Vol. 4, No. 1, 2003, pp. 625-632. doi:10.1.1.460.2814.

[40] Rouse, H., and Abul-Fetouh, A., "Characteristics of irrotational flow through axially symmetric orifices," Journal of Applied Mechanics-Transactions of the ASME, Vol. 17, No. 4, 1950, pp. 421-426.

[41] Lamb, H., Hydrodynamics, Cambridge university press, 1993. 